\title{
Feature Extraction and Pupil Detection Algorithm Used for Iris Biometric Authentication System
}

\author{
Vanaja Roselin E. Chirchi ${ }^{1}$ and L. M. Waghmare ${ }^{2}$ \\ ${ }^{1}$ Research Scholar, JNTUH, Kukatpally Hyderabad-500085(AP), India \\ ${ }^{2}$ Director, SGGS Institute of Engineering and Technology \\ Vishnupuri, Nanded-431606(MS), India \\ vr.chirchi@gmail.com
}

\begin{abstract}
Iris biometric is most mature and secure authentication system as compared to other systems. Other authentication system does exist apart from biometrics such as PIN, password etc., which are not secure and more vulnerable to attacks and can be hacked or spoofed easily. Using, Iris biometric we can enhance overall performance in terms of accuracy, which is possible if and only if the pupil part must be removed perfectly and efficiently. Pupil part is unwanted part for our system. Pupil part is surrounded by iris part when extracted successfully we get two approximate concentric circle. Proposed scanning algorithm successfully extracts pupil part and defines iris part with less complexity and more efficiently. Iris part is consisting of patterns which are desired for authenticating a person and each patterns are represented in terms of feature vectors and stored in database. Proposed system focuses on feature extraction using five level decomposition technique implemented with haar, db2 and $d b 4$ and achieves high accuracy with reduced error rates. Due to reduced errors and considering lower half of iris part, proposed algorithm can be used for larger database such as for Aadhar because it takes less time for feature extraction and has less complexity with reduced mathematical burden on the system and improves good accuracy.
\end{abstract}

Keywords: Iris biometrics; pupil extraction; feature extraction; false acceptance Rate (FAR); False Rejection Rate (FRR); Equal Error Rate (EER)

\section{Introduction}

\subsection{Overview of Biometrics in Security Systems}

Biometrics, which refers to authentication based on his or her physiological or behavioral characteristics, its capability to distinguish authorized person and an unauthorized. Since biometric characteristics are distinctive as it cannot be forgotten or it cannot be lost, for identification, person has to be present physically. Biometric is more reliable and capable than traditional knowledge based and token-based techniques. Biometric has also drawback i.e., if it is compromised then it is difficult to replace. Among all biometrics such as fingerprint, facial thermogram, hand geometry, face, hand thermogram, iris, retina, voice, signature etc., Iris-based Recognition is one of the most mature and proven technique. Iris is colored part of eye as in Figure 1.1. A person's two eye iris has different iris pattern, two identical twins also has different in iris patterns because iris has many feature which distinguish one iris from other, primary visible characteristic is the trabecular meshwork, a tissue which gives the appearance of dividing the iris in a radial fashion that is permanently 
formed by the eighth month of gestation [27] and iris is protected by eyelid and cornea as shown in Figure 1 therefore increases security of the systems. Spoofing is very difficult with iris patterns as compare to other biometrics. In practical situation it is observed that iris part is occluded by interference of eyelids and eyelashes, improper eye opening, light reflection and image quality is degraded because of low contrast image and other artifact [14]. Advantages of Iris is that it is not subject to the effects of aging which means it remains in a stable form from about age of one until deat. The use of glasses or contact lenses has little effect on the representation of the iris and hence does not interfere with the recognition technology [27].

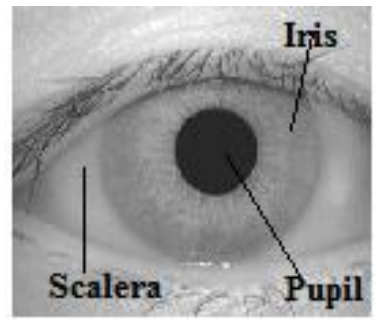

\section{Figure 1. Structure of Iris}

\subsection{Iris Enrollment}

Iris-based Recognition is one of the most mature and proven technique. This section gives details of the proposed system as in Figure 2. System is classified into enrollment process and authentication process. Enrollment process is the process of capturing information of the subject and storing them in to database $(\mathrm{db})$. Enrollment process consists of iris image acquisition, pupil detection and iris localisation, normalization, extraction of feature from iris image. These features are stored in the form of Iriscode in database (db).

\subsection{Iris Recognition / Verification}

Recognition or verification process compares the input features with enrolled feature in the systems database $(\mathrm{db})$ with a reference. Performance improvement of iris recognition system is possible with reduced false rejections, false acceptance and equal error rate. The system is consisting of 5 steps process to achieve the results. Therefore systems steps are as follows:

Step1: Image Acquisition: It is the process of acquiring image, which is done using CCD camera. Acquiring images of Iris is major aspect for the recognition system. Images with good resolution and sharpness need to maintain with adequate intensity. Our research uses publicly available Iris database from Institute of automation, Chinese academic of science (CASIA) with 756 grayscale images of eye with 108 unique eyes or classes and seven different images of each eye.

Step2: Iris localization: when eye is captured in CCD camera, next need to acquire only iris pattern, extracting pupil part.

Step3: Iris Normalization: After extracting pupil achieve circular iris, which is to be converted to rectangular form.

Step 4: Feature Extraction: Decomposing and formation of iris pattern into iris codes.

Step 5: Matching or Verification: accept or reject by comparing stored enrolled pattern of database with submitted pattern. 


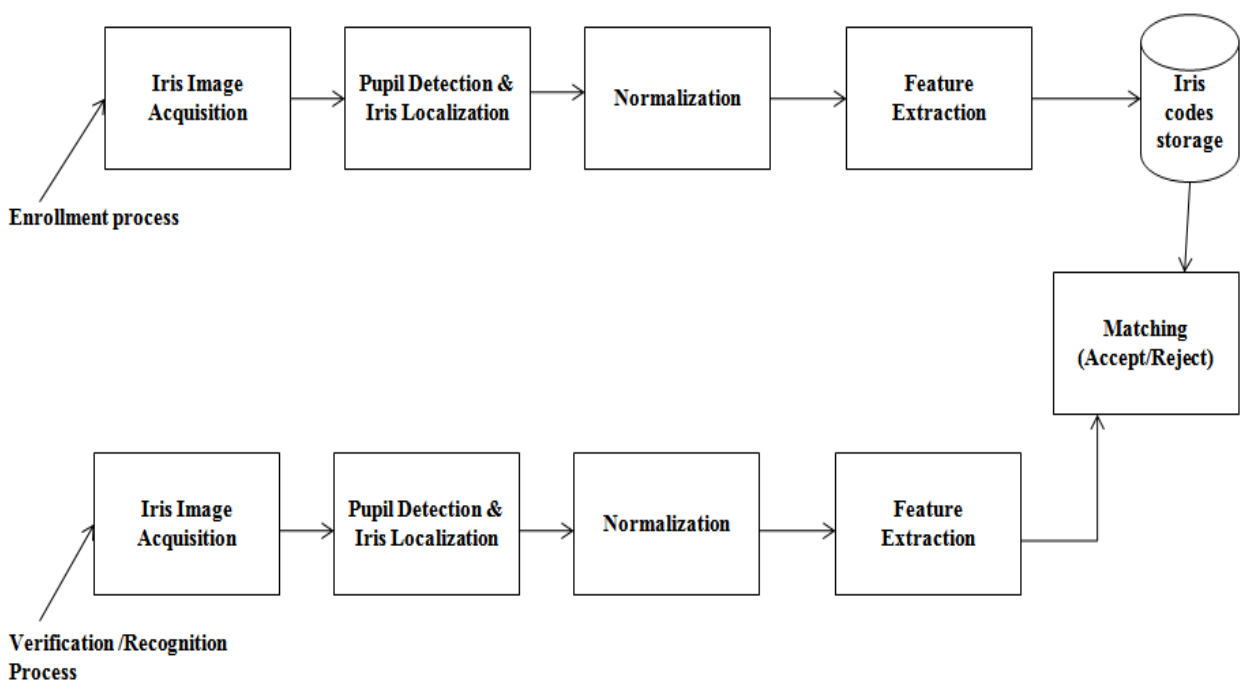

Figure 2. Overview of Iris Recognition System Model

\subsection{Overview}

Pupil is surround by colored part called Iris as in Figure 1, which is unwanted part for our experiment, so pupil extraction is performed using scanning method which is simple in terms of computational complexity cost and thus reduces mathematical burden on the system, achieves high accuracy, increased correct recognition rate and reduced time for overall system performance. Once pupil is extracted, iris is located and iris texture is considered as iris feature which are then analyzed for person recognition. Feature extraction is performed using five level decomposition technique using haar wavelet and daubechies wavelet. And later perform comparision between wavelets and this shows that haar wavelet achieves better results than daubechies ( $\mathrm{db} 2$ and $\mathrm{db} 4)$. After implementation considering haar wavelet as best suited for feature extraction. To extract feature of image, image is passed with low pass filter and high pass filter with down sample factors and thus decompose up to five levels, which results as decomposed image with 90 feature vector elements. These features elements are clear and sufficient to perform person identification and recognition efficiently.

\section{Literature Review}

Various approaches exist in the past for iris recognition for person identification which includes John Daugman's Iriscode [5]. However proposed work uses scanning method for pupil detection and iris localization and five level decomposition techniques for haar wavelet for iris feature extraction to get 90 feature vector elements for effective iris recognition. Advantages of proposed methods are its computational simplicity and speed. This method is less likely to be affected by environmental factors as compared to Gabor wavelet The Iris Recognition system's main work role is to provide compact and significant feature extraction algorithm for iris images with reduced false rejection rate. The extracted feature should have high discriminating capability and the segmented iris image should be free from artifacts [1]. Daugman [5] used Integro-differential operator for pupil detection and a multiscale quadrature two-dimentional (2-D) Gabor filter to demodulate phase information of an iris image to create an Iriscode for authentication by comparing the Iriscode stored in database. Li.Ma et al., [15] used Hough transformation and extracted features using spatial filter, this technique first converts the round image of the iris into rectangular pattern by unwrapping the 
circular image. Wildes et al., [21] uses Hough transform and gradient edge detection for pupil detection and Laplacian pyramid for analysis of the Irisimages. Boles and Boashash [29] uses zero-crossing method with dissimilarity functions of matching. Lim et al., [25] 2D Haar Transform for feature extraction and classifier used are initialization method of the weight vectors and a new winner selection method designed for iris recognition. A. Poursaberi and H. N. Araabi [1, 2] use wavelet Daubechies2 for feature extraction and two classifiers such as Minimum Hamming Distance and Harmonic mean. Li. Ma et al., [14] class of 1-D wavelet i.e., 1-D Intensity signals for feature extraction and for feature matching they have used expanded binary feature vector with exclusive OR operations. Md. Rabiul Islam et al., [19] used 4-level db8 wavelet transform for feature extraction and hamming distance with XOR for pattern matching. In our proposed research work will be using wavelets such as Haar, db2, and $\mathrm{db} 4$ for feature extraction and perform comparison on the basis of their performance evaluation. Use Hamming Distance classifier to matching binary strings with enrolled entity in the database. To fasten the matching speed, a lower number of bits, i.e., 348 bits is used in composing the iris code, as compared with other methods such as 2048 bits in [4,5]. Comparision of iris feature extraction and classifier algorithm are as shown in Table 1.

\section{Table 1. Methodologies used by Researchers for Pupil Detection and Feature Extraction}

\begin{tabular}{|c|c|c|c|c|c|}
\hline $\begin{array}{l}\text { Sl. } \\
\text { No. }\end{array}$ & $\begin{array}{l}\text { Researcher's } \\
\text { methods }\end{array}$ & $\begin{array}{c}\text { Feature } \\
\text { Extraction }\end{array}$ & $\begin{array}{l}\text { Matching } \\
\text { Process }\end{array}$ & $\begin{array}{l}\text { Feature } \\
\text { vector }\end{array}$ & Results \\
\hline 1 & Daugman[5] & 2D Gabor & $\begin{array}{l}\text { Hamming } \\
\text { Distance with } \\
\text { XOR }\end{array}$ & $\begin{array}{l}\text { Binary i.e., } 2048 \text { bit } \\
\text { phase vector }\end{array}$ & $\begin{array}{l}300 \mathrm{MHZ} \text { CPU, } \\
\text { search are performed } \\
\text { at the rate of about } \\
100,000 \text { iris per } \\
\text { second. }\end{array}$ \\
\hline 2 & Wildes [21] & $\begin{array}{l}\text { Laplacian pyramid } \\
\text { \& Gaussian Filters }\end{array}$ & $\begin{array}{l}\text { Normalized } \\
\text { Hamming } \\
\text { Distance with } \\
\text { exclusive OR } \\
\text { operator }\end{array}$ & 256 bytes & - \\
\hline 3 & $\begin{array}{l}\text { A.Poursaberi } \\
\text { \& H.N. } \\
\text { Araabi[1][2] }\end{array}$ & $\begin{array}{l}\text { Wavelet Based } \\
\text { Feature extraction }\end{array}$ & $\begin{array}{l}\text { Minimum } \\
\text { Hamming } \\
\text { Distance(MHD) \& } \\
\text { Harmonic mean }\end{array}$ & $\begin{array}{l}408(544) \text { binary } \\
\text { feature vector }\end{array}$ & $\begin{array}{l}\text { CRR is } 99.31 \% \text { \& } \\
\text { ERR is } 0.2687 \%\end{array}$ \\
\hline 4 & $\begin{array}{l}\text { Vatsa et } \\
\text { al.,[18] }\end{array}$ & $\begin{array}{l}\text { 1-D log polar Gabor } \\
\text { Transform \& } \\
\text { Topological feature } \\
\text { extraction using } \\
\text { Euler No. }\end{array}$ & $\begin{array}{l}2 \mathrm{v}-\mathrm{SVM} \text { method } \\
\text { for matching the } \\
\text { texture } \& \\
\text { topological } \\
\text { features }\end{array}$ & - & $\begin{array}{l}\text { Performance in terms } \\
\text { of accuracy is } \\
97.21 \%\end{array}$ \\
\hline 5 & $\begin{array}{l}\text { Makram } \\
\text { Nabti et } \\
\text { al.,[17] }\end{array}$ & $\begin{array}{l}\text { Wavelet maxima } \\
\text { component as } \\
\text { multiresolution } \\
\text { technique \& special } \\
\text { Gabor filter bank }\end{array}$ & $\begin{array}{l}\text { Hamming } \\
\text { Distance with } \\
\text { XOR }\end{array}$ & $\begin{array}{l}\text { Statistical feature } \\
\text { with } 480 \text { vector } \\
\text { elements } \& \text { moments } \\
\text { invariants using } \\
1680 \text { vector elements }\end{array}$ & $\begin{array}{l}\text { Feature extraction } \\
\text { computational } \\
\text { complexity (ms), } \\
\text { statistical feature: } \\
74, \text { Moment } \\
\text { invariants: } 81\end{array}$ \\
\hline 6. & $\begin{array}{l}\text { Amol D. } \\
\text { Rahulkar } e t \\
\text { al.,[3] }\end{array}$ & $\begin{array}{l}\text { Biorthogonal Triplet } \\
\text { Half Band Filter } \\
\text { Bank(THFB) }\end{array}$ & $\begin{array}{l}\text { Flexible k-out-of- } \\
\text { n: postclassifier }\end{array}$ & $\begin{array}{l}7 \text { integer values per } \\
\text { region }\end{array}$ & $\begin{array}{l}\text { Low computational } \\
\text { complexity with } \\
\text { significant reduced } \\
\text { FRR. }\end{array}$ \\
\hline 7 & $\begin{array}{l}\operatorname{Lim} e t \\
\text { al.,[25] }\end{array}$ & $\begin{array}{l}\text { Haar wavelet } \\
\text { Transform }\end{array}$ & $\begin{array}{l}\text { LVQ neural } \\
\text { network }\end{array}$ & $\begin{array}{l}87 \text { dimensions } \\
\text { (1 bit/dimension) } \\
\text { i.e.,87bits }\end{array}$ & $\begin{array}{l}\text { Recognition } \\
\text { performance is } \\
98.4 \%\end{array}$ \\
\hline
\end{tabular}




\begin{tabular}{|l|l|l|l|l|l|}
\hline 8 & $\begin{array}{l}\text { L. Ma } \text { et } \\
\text { al.,[14] }\end{array}$ & $\begin{array}{l}\text { Class of 1-D } \\
\text { Wavelets i.e., 1-D } \\
\text { Intensity signals }\end{array}$ & $\begin{array}{l}\text { Expanded binary } \\
\text { Feature vector \& } \\
\text { Exclusive OR } \\
\text { operations }\end{array}$ & $\begin{array}{l}\text { Vector consists of } \\
660 \text { components \& } \\
\text { represented in byte. }\end{array}$ & $\begin{array}{l}\text { CRR is 100\% \& } \\
\text { EER is 0.07\% \& } \\
\text { computational } \\
\text { complexity is } \\
\text { 250.7(ms) }\end{array}$ \\
\hline 9 & $\begin{array}{l}\text { Md. Rabiul } \\
\text { Islam } \text { et } \\
\text { al.,[19] }\end{array}$ & $\begin{array}{l}\text { 4-level db8 wavelet } \\
\text { transform }\end{array}$ & $\begin{array}{l}\text { Hamming } \\
\text { Distance with } \\
\text { XOR }\end{array}$ & $\begin{array}{l}\text { Binary codes of 510 } \\
\text { bits }\end{array}$ & $\begin{array}{l}\text { CRR is 98.14\% \& } \\
\text { ERR is } 0.21 \%\end{array}$ \\
\hline 10 & $\begin{array}{l}\text { Proposed } \\
\text { Method }\end{array}$ & $\begin{array}{l}\text { 5-level Wavelet } \\
\text { transformation } \\
\text { method such as } \\
\text { Haar,db2,db4 }\end{array}$ & $\begin{array}{l}\text { Hamming } \\
\text { Distance with } \\
\text { XOR }\end{array}$ & FV of 90 bits & $\begin{array}{l}\text { EER=0.03\% } \\
\text { CRR=99.97\% }\end{array}$ \\
\hline
\end{tabular}

\section{Formation of the Problem and Methodology}

\subsection{Objectives}

\section{Objective of our research is to}

(a) Enhancing pupil detection algorithm for efficient and fast with less mathematical burden on system.

(b) To perform feature extraction efficiently even though upper portion of the eye is densely covered by eyelashes.

(c) To improve overall performance of the system and achieve accuracy with minimized error rates.

\subsection{Contribution of Thesis}

Research problem is enhancement of person identification and recognition by reducing complex mathematical burden and simplifying system complexity cost in terms of time. The contribution to the research is at pupil detection phase using scanning method and at feature extraction using five level decomposition techniques applied to algorithms. In this research, we are going to resolve the problem of efficiency using pupil detection by scanning method which is simple and fast and feature extraction using 5-level wavelet decomposition technique.

\subsection{Materials and Methods}

Our implementation is performed using Matlab7.0 on PC with $2.00 \mathrm{GHz}$ dual processor and 1.00 GB RAM.

\section{Pupil Detection and Iris Localisation}

\subsection{Dataset}

Database used for system is CASIA Iris database with training image of 756 iris images and testing images are 100 .

\subsection{Algorithm}

Scanning method is the proposed method for pupil detection. Pupil is a dark part of eye. Consider pupil and iris as two concentric circles. Histogram is graphical representation of 
image with number of pixel on y-axis and pixel intensity on $x$ - axis, plot histogram for input image. Histogram extracted from image as it is made up of bins, each bin representing a certain intensity value range. It is computed by examining all pixels in the image and assigning each to a bin depending on pixel intensity. The value of bin is no of pixel assigned to it. Our method is simple with minimized complexity with less mathematical burden to the system, which is well suited for other database such as MMU, UBIRIS. Daugman [5] uses Integro differential operator which has mathematical burden to system, Wilde et al., [21] uses gradient based edge detection which is complex, Poursaberi and Araabi [1] uses image morphological operator and suitable threshold with more calculation. Proposed algorithm as follows:

Step1: Read the original image from database as shown in Figure 1.

Step2: Draw Histogram for iris image and calculate threshold value of pixel intensity. As shown in Figure 3.

Step3: Mark and fix LF(left) pixel point as start point on $\mathrm{x}$-axis and begin scanning on $\mathrm{x}$-axis, as pupil is dark part of the eye, Assign dark pixel with value as 0 and grey pixel is end of the dark pixel mark and fix as RT(Right) pixel point, assign value as 1, as in Figure 4.

Step4: Similarly Mark and Fix UT (UpperTop) pixel point and scan on y-axis, assign dark pixel value as 0 and where the dark ends mark and fix as LB (LowerBottom) pixel assign the value as 1, as in Figure 4.

Step5: To locate center $\mathrm{C}$ of pupil, calculate $\mathrm{C}=[(\mathrm{LF}+\mathrm{RT}) / 2,(\mathrm{UT}+\mathrm{LB}) / 2]$

Step6: Determining pupil radius (PR)

PR1 $=$ abs $($ RT $-\mathrm{C})$

$\mathrm{PR} 2=\operatorname{abs}(\mathrm{C}-\mathrm{LF})$

PR3 = abs (UB-C)

PR4= abs(C-UT)

Pradius_array [PR1, PR2, PR3, PR4]

$\mathrm{PR}=$ max [Pradius_array]

Once pupil radius is calculated, add 38 pixels to it, so results with Iris radius, i.e.,

Iris Radius = Pupil_radius $(\mathrm{PR})+38$ defined in [1]. Once pupil radius and iris radius is known then form the approximate concentric circle as in Figure 5.

Calculating threshold is major aspect and depends on pixel intensity. Pupil is black color; pixel intensity lies closer to zero and gray levels in the range of 0 to 50.

Algorithm removes pixel having less number of counts than threshold.

Step1: Determine the connected component of binary image (bw)

Step2: Computing area of each component of binary image, i.e., region of bw.

Step3: Remove undesirable objects less than threshold and store.

The formula for threshold (T) of image is as in eq 1.

$$
\begin{aligned}
& \mathrm{g}(\mathrm{m}, \mathrm{n})=1 \text { if }(\mathrm{m}, \mathrm{n}) \leq \mathrm{T} \\
& \qquad \mathrm{g}(\mathrm{m}, \mathrm{n})=0 \text { if }(\mathrm{m}, \mathrm{n}) \geq \mathrm{T}
\end{aligned}
$$

Where $\mathrm{g}(\mathrm{m}, \mathrm{n})$ is gradient vector of image. 


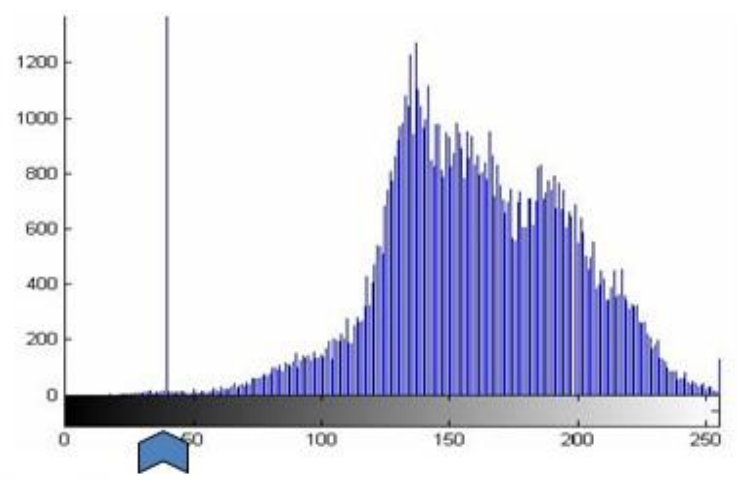

\section{Pupil intensity}

As threshold value

Figure 3. Histogram for Input Image

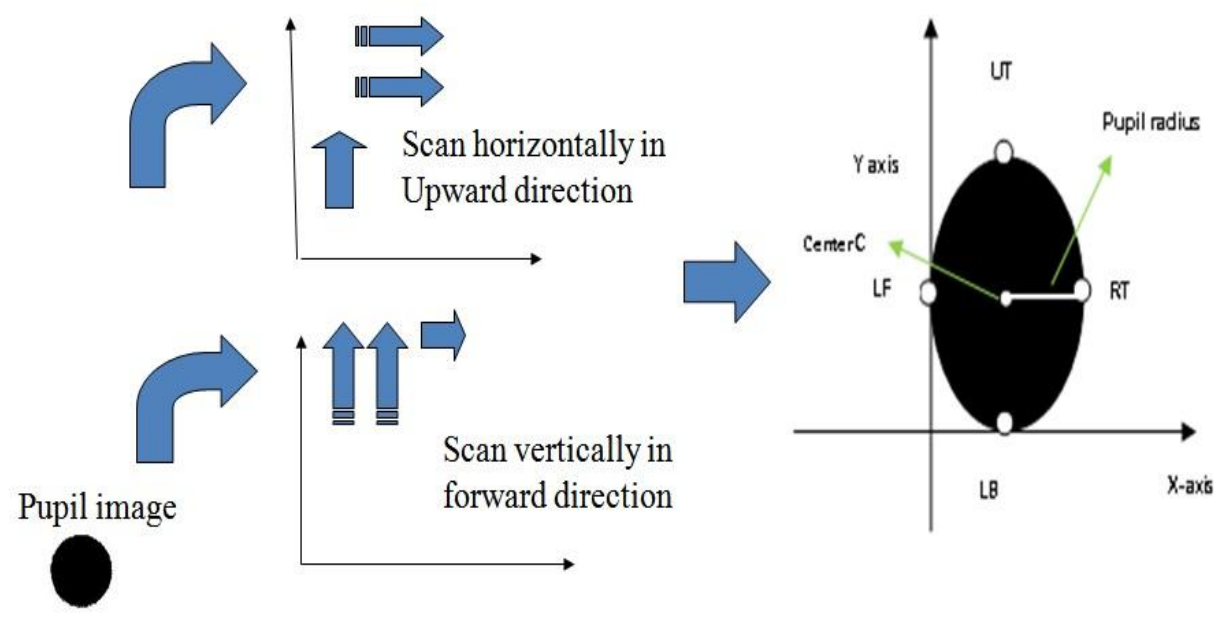

Figure 4. Scanning and Locating Four Coordinate Points on Pupil Circle

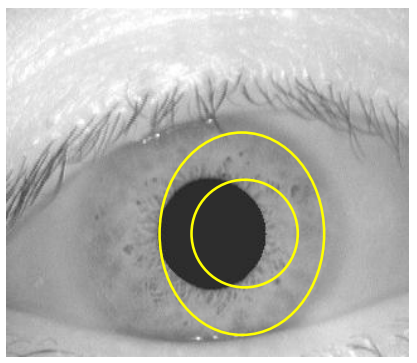

Figure 5. Locating Iris and Pupil Circle

\subsection{Iris Localization}

Consider the iris radius (as in eq2) [1].

Iris_radius $=$ pupil_radius +38

Where 38 pixel elements are defined in [1], add this to pupil radius to obtain Iris radius. Thus pupil is extracted from input image and iris is located, which is used for further processing. 
To draw the circle from pupil boundary to iris boundary using canny edge detection algorithm which results with two approximate concentric circles as shown in Figure 5.

\subsection{Results}

Proposed method shows significant and efficient results in terms of speed as compared to Daugman [6] and Wildes [21] as in Table 2, implemented in Matlab 7. 0 on PC with 2.00 $\mathrm{GHz}$ processor and $1.00 \mathrm{~GB}$ RAM. Figure 6 is a comparative graph of renowned authors whose system has perfect accuracy and speed but proposed method shows better results as in Table 2.

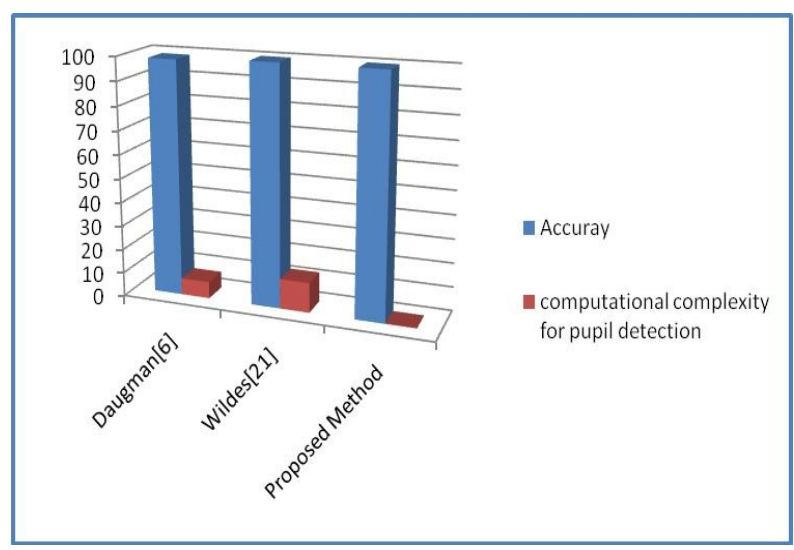

Figure 6. Comparative Graph

Table 2. Computational Complexity Cost for Pupil Detection

\begin{tabular}{|c|c|c|}
\hline Methods & Accuracy (\%) & Computation complexity(Secs) \\
\hline Daugman[6] & 98.6 & 6.99 \\
\hline Wildes[21] & 99.9 & 12.54 \\
\hline Proposed Method & 99.97 & 0.32 \\
\hline
\end{tabular}

\subsection{Conclusion}

Due to proposed method computational cost has been achieved significantly and efficiently in terms of speed when compared with other authors as in Table 2. For this achievement the system configuration used is PC with $2.00 \mathrm{GHz}$ processor and $1.00 \mathrm{~GB}$ ram and implemented in Matlab 7.0. Comparative graph of renowned authors whose system has perfect accuracy and speed but proposed method shows better results.

\section{Normalization}

\subsection{Introduction}

- Use of Daugman's rubber sheet model.

- Representing Cartesian to polar coordinates.

- Output as normalized iris image. 


\subsection{Implementation}

Consider iris part for further processing after pupil is successfully extracted. In our research lower half part of iris is considered because most of the time upper iris section is densely covered by the eyelashes which can affect system performance and decreases the accuracy of overall system. Consider coordinate system and unwrap the lower part of the iris i.e., lower 180 degree. Map all the points within the boundary of the iris into their polar equivalent using Daugman's rubber sheet model as shown in Figure 7. The process is achieved to be a standard form irrespective of iris size, pupil diameter or resolution. Working idea of the dimensionless polar system is to assign 32 pixels along $r$ and 180 pixels along $\Theta$ value to each coordinate in the iris that will remain invariant to the possible stretching and skewing of the image and results with unwrapped strip of 32 X 180 sizes. Thus the process gives us the normalized image as in Figure 8.

The remapping of the iris image $I(\mathrm{x}, \mathrm{y})$ from raw Cartesian coordinate to polar coordinates $I(\mathrm{r}, \theta)$ can be represented as in eq 2 .

$$
I(\mathrm{x}(\mathrm{r}, \theta), \mathrm{y}(\mathrm{r}, \theta)) \longrightarrow(\mathrm{r}, \theta)
$$

Where $\mathrm{r}$ radius lies in the unit interval $(0,1)$ and $\theta$ is the angle between $(0,2 \pi)$ for Iris image $I$

The eq. 3 yields from eq. 3 and eq. 4 and they are

$$
\begin{aligned}
& x(r, \theta)=(1-r)^{*} x_{p}(\theta)+r^{*} x_{i}(\theta) \\
& y(r, \theta)=(1-r)^{*} y_{p}(\theta)+r^{*} y_{i}(\theta)
\end{aligned}
$$

Where $\left.\left(\mathrm{x}_{\mathrm{p}}(\theta)\right), \mathrm{y}_{\mathrm{p}}(\theta)\right)$ and $\left(\mathrm{x}_{\mathrm{i}}(\theta), \mathrm{y}_{\mathrm{i}}(\theta)\right)$ are the coordinates of pupil and iris boundary points respectively.

The normalization step not only reduces exactly the distortion of the iris caused by pupil movement and also simplifies subsequent processing [22].

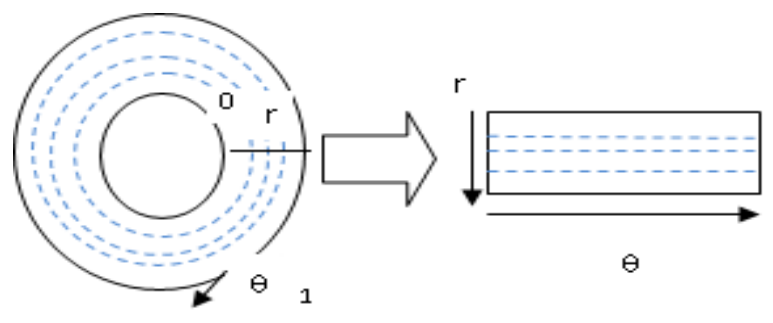

Figure 7. Daugman's Rubber Sheet Model with Annular Iris Zone is Stretched to a Rectangular Block and Dashed Lines are Sampling Circles 


\section{Original image}
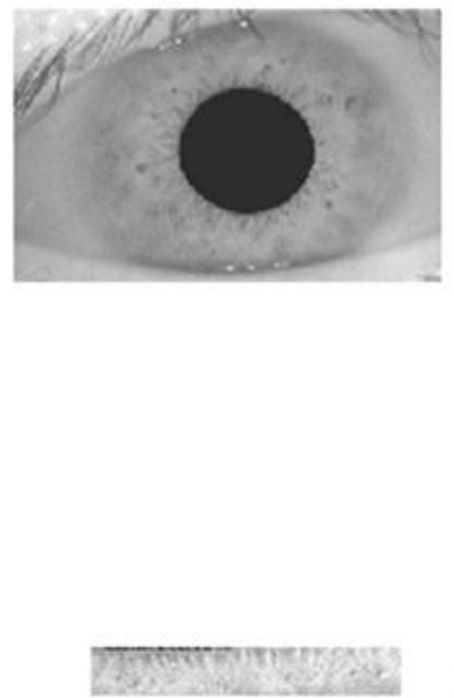

Normalized iris 32 X 180 Size

\section{Pupil image}
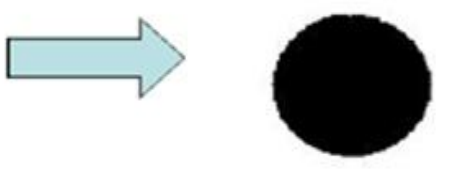

Segmented

iris
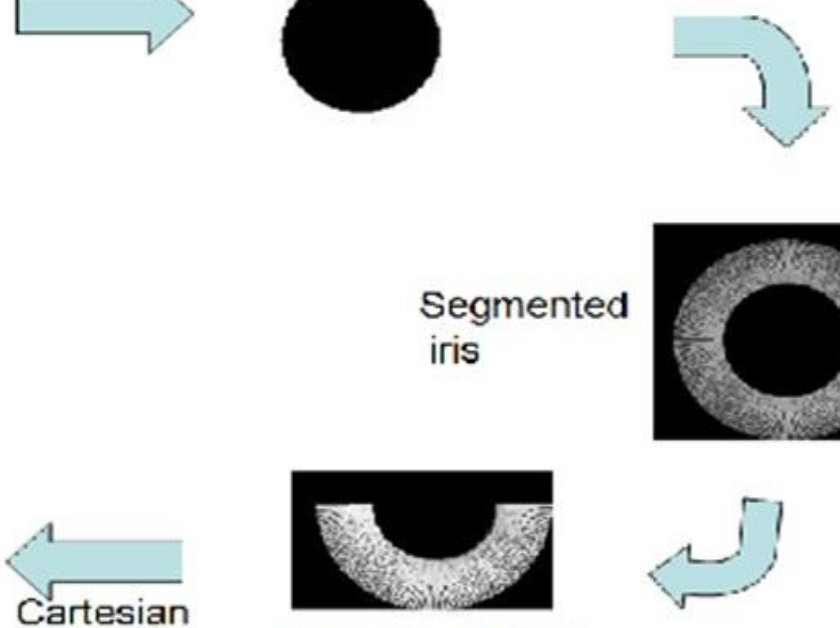

To polar

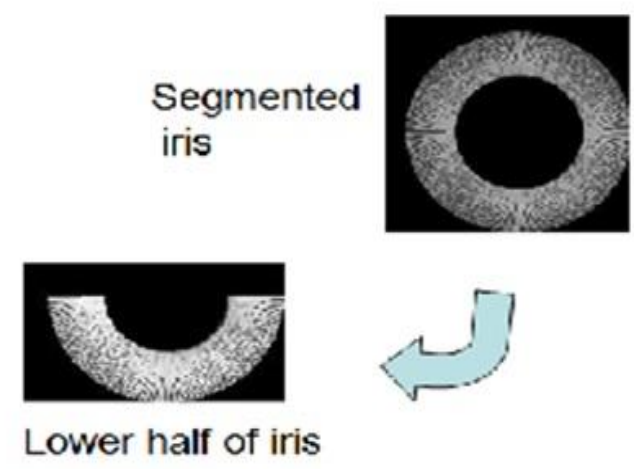

Figure 8. Normalization Process

\subsection{Conclusion}

With Daugman's rubber sheet model, representation of Cartesian to polar coordinates is successful. System achieved desired unwrapped strip of size 32X180, which is sufficient for accurate and fast person recognition.

\section{Feature Encoding and Matching}

\subsection{Introduction}

The iris has abundant texture information for accurate recognition of individual. Extract the pattern of the iris image with out noise so that quality of matching will be enhanced. Proposed system consider Haar wavelet and daubenchies wavelet(db2 and db4) for extracting feature using five level decomposition techniques.

The following steps for feature extraction.

1. Apply 2D DWT with Haar and Daubenchies( db2 and db4) up to 5-level decomposition.

2. Using $4^{\text {th }}$ level, $5^{\text {th }}$ level decomposition details constructed the feature vectors.

3. Feature vectors are in the form of Iriscodes(binary form).

4. Store these feature vectors in database $(\mathrm{db})$. 


\subsection{Block Diagram of Feature Extraction}

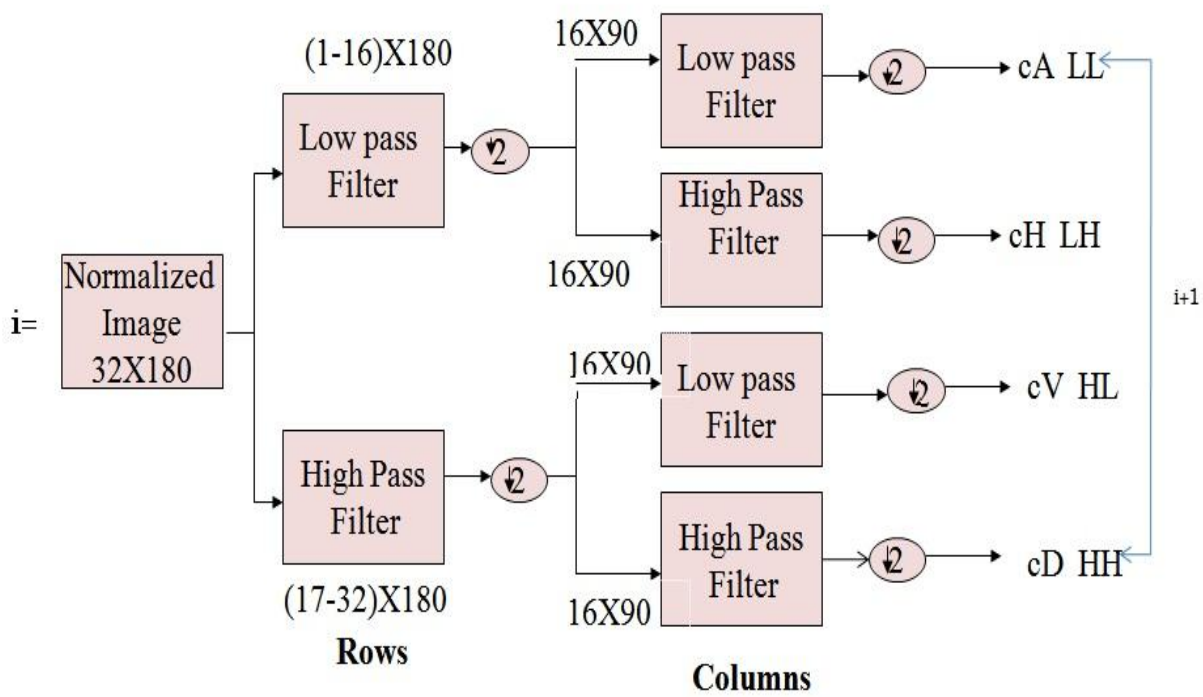

LL(Low to Low), LH(Low to High), HL(High to Low), HH(High to High)

cA: Approximation coefficient matrix

cH,cV,cD: Detail coefficient in terms of(Horizontal, vertical, diagonal)

Figure 9. Block Diagram of Decomposition Technique for Feature Extraction

Figure 9 shows the decomposition of the image with down sample of 2 and storing in approximation $(\mathrm{cA})$ and detail $(\mathrm{cH}, \mathrm{cV}, \mathrm{cD})$ matrices.

\subsubsection{Implementation with five level decomposition Technique(Proposed)}

Why five level decomposition Technique? Because decomposing images with a wavelet transform yields a multi-resolution from detailed image to approximation image in each levels considering image of size $\mathrm{N} X \mathrm{M}(320 \mathrm{X} 280)$ and decompose up to $\mathrm{K}^{\text {th }}$ level where $\mathrm{K}=1,2,3,4,5$. The quadrants (sub images) with in images as the $\mathrm{LH}$ (Low pass filter to High pass filter), HL(High pass filter to Low pass filter) and $\mathrm{HH}$ (High pass filter to High pass filter) represents detailed, i.e., images for horizontal, vertical and diagonal orientation in the first level. The sub images LL (Low pass filter to Low pass filter) corresponds to an approximation image that is further decomposed, resulting in further decomposed image which level two. Obtain $5^{\text {th }}$ level wavelet tree showing all detail and approximation coefficients these levels are $\mathrm{CV}_{1}$ to $\mathrm{CV}_{5}$ (vertical coefficient), $\mathrm{CH}_{1}$ to $\mathrm{CH}_{5}$ (horizontal coefficient), $\mathrm{CD}_{1}$ to $\mathrm{CD}_{5}$ (diagonal coefficient). After $5^{\text {th }}$ level, combine vertical, Horizontal and Diagonal coefficients of $4^{\text {th }}$ level and $5^{\text {th }}$ level, i.e., LH4, HH4, HL4, LH5, HH5, HL5 obtains feature vector of 90 elements because contains image pixel information which are sufficient for person identification efficiently and decomposition steps are as follows.

- Step 1: Input normalized image, i.

- Step 2: Consider rows blocks, call Low Pass Filter (LPF ()) and High Pass Filter (HPF ()) functions.

- Step 3: Down sample columns by 2 and Keep even index columns. 
- Step 4: Consider column blocks, call LPF () and HPF () functions.

- Step 5: Down sample Rows by 2 and keep even index rows.

- Step 6: Convolve Rows and Columns of filter entries.

- Step 7: Store in Approximation matrix coefficient and Detail matrix coefficient in term of Low to Low (LL) for approximation, Low to High (LH) for Horizontal, High to Low(HL) for vertical and High to High(HH) for Diagonal.

- Step 8: Output Decomposed image for level 1.

- Step 9: Repeat step 2 to step 7 for i+1 image and decompose image for Level2, level3, level4, level 5.

Figure 11 shows the results of first level decomposed image with coefficient such as approximation coefficient, first horizontal coefficient, vertical coefficient and diagonal coefficient, size of the first decomposed images are 16 X 90 pixels. Similarly obtain second level decomposition approximation, horizontal, vertical, diagonal details has the size 8 X 45 . In third level decomposition approximation, horizontal, vertical, diagonal details have size 4 $X$ 23. In forth level decomposition approximation, horizontal, vertical, diagonal details have size 2 X 12. In fifth level decomposition approximation, horizontal, vertical, diagonal details have size $1 \mathrm{X} 6$. Now pick up the coefficients that represent the core of the iris pattern. Therefore those that reveal redundant information should be eliminated. In fact, it is obvious that the patterns in the $c D_{1}{ }^{h}, c D_{2}{ }^{h}, c D_{3}{ }^{h}, c D_{4}{ }^{h}$, are almost the same and only one can be chosen to reduce redundancy. Since $c D_{4}^{h}$ repeats the same patterns as the previous horizontal detail levels and it is the smallest in size, then take it as a representative of all the information the four levels carry. The fifth level does not contain the same textures and should be selected as a whole. In a similar fashion, only the fourth and fifth vertical and diagonal coefficients can be taken to express the characteristic patterns in the iris-mapped image. Thus represents each image applied to the Haar wavelet as the combination of six matrices i.e. $c D_{4}{ }^{h}, c D_{5}{ }^{h}, c D_{4}{ }^{v}$, $c D_{5}{ }^{v}, c D_{4}{ }^{d}$ and $c D_{5}{ }^{d}$. These matrices are combined to build one single vector characterizing the iris patterns. Such vector is called Feature vector. Since all mapped images have a fixed size of 320 X 280 then all images will have a fixed feature vector. In our proposed work consider the vector size of 90 elements as shown in conceptual diagram of Figure 10 (a) and (b).
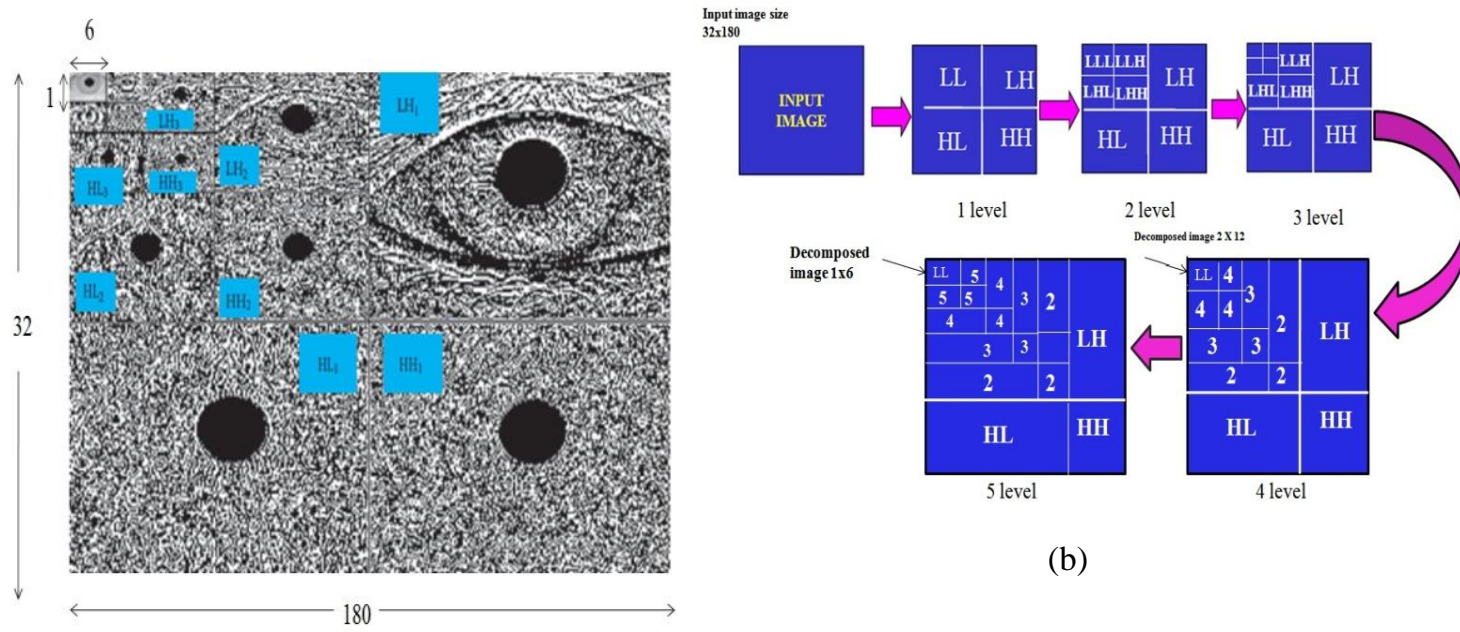

(b)

(a)

Figure 10. (a ) and (b ) Conceptual Diagram 


\subsubsection{Feature Encoding}

After achieving feature vector, need to represent it in a binary code as it is easy to make the difference between two binary code words than between two number vectors. Thus Boolean vectors are always easier to compare and easier to manipulate. After observing characteristic, code the feature vector by considering the condition (as in eq. 5) results the vectors have maximum value greater than 0 and a minimum value that is less than 0 i.e., if Coef is the feature vector of an image than the following quantization scheme converts it to its equivalent code word

$$
\begin{aligned}
& \text { If Coef (i) } \geq 0 \text { then Coef (i) }=1 \\
& \text { If Coef }<0 \text { then Coef (i) }=0
\end{aligned}
$$

After representing in binary coding scheme, need to match the two codes to check whether it belongs to same person or not.

\subsection{Results}

Our approach considers Haar, $\mathrm{db} 2, \mathrm{db} 4$ and found haar is more efficient with minimum feature vector as compared to db2 and db4 as in Table 3. Experiment is carried using haar wavelet with 5-level decomposition for feature extraction. Figure 10 shows the conceptual diagram for organizing feature vector by five level decomposition of normalized image. Logical diagram is as shown in Figure 9.
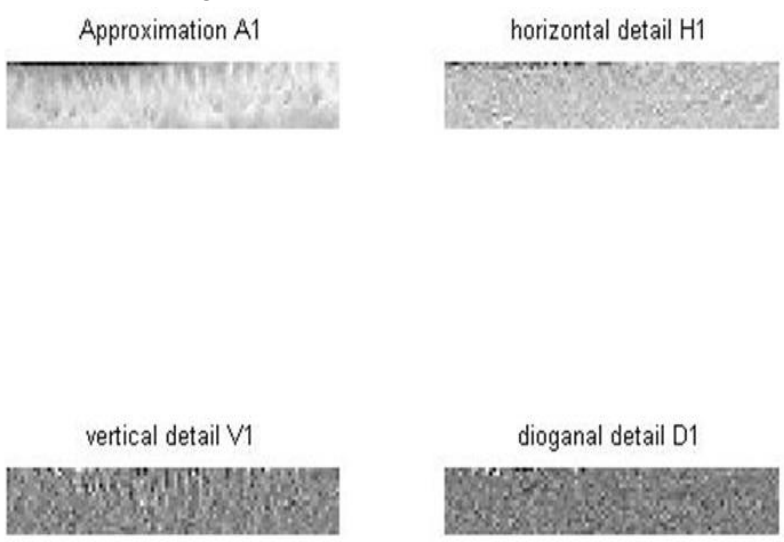

Figure $11.1^{\text {st }}$ level haar decomposition in terms of cA1, cV1, cH1, cD1

Table 3. Feature vector of wavelet

\begin{tabular}{|c|c|c|c|c|c|c|c|}
\hline $\begin{array}{c}\text { Wavelet } \\
\text { algorithm }\end{array}$ & $\begin{array}{c}\text { Normalized } \\
\text { image size }\end{array}$ & $\begin{array}{c}\text { cA1,cH1 } \\
\text { cV1,cD1 }\end{array}$ & $\begin{array}{c}\text { cA2,cH2 } \\
\text { Cv2,cD2 }\end{array}$ & $\begin{array}{c}\text { cA3,cH3 } \\
\text { cV3,cD3 }\end{array}$ & $\begin{array}{c}\text { cA4,cH4 } \\
\text { cV4,cD4 }\end{array}$ & $\begin{array}{c}\text { cA5,cH5 } \\
\text { cV5,cD5 }\end{array}$ & $\begin{array}{c}\text { Feature } \\
\text { Vector }\end{array}$ \\
\hline Haar & $32 \times 180$ & $16 \times 90$ & $8 \times 45$ & $4 \times 23$ & $2 \times 12$ & $1 \times 6$ & $1 \times 90$ \\
\hline $\mathrm{Db} 2$ & $32 \times 180$ & $17 \times 91$ & $10 \times 47$ & $6 \times 25$ & $4 \times 14$ & $3 \times 8$ & $1 \times 240$ \\
\hline $\mathrm{Db} 4$ & $32 \times 180$ & $19 \times 93$ & $13 \times 50$ & $10 \times 28$ & $8 \times 17$ & $7 \times 12$ & $1 \times 660$ \\
\hline
\end{tabular}

\subsection{Conclusion}

The cost for computational complexity in milliseconds is best achieved with decomposition technique of image up to five levels using 2D Haar wavelet and it is fast as compared to other methods for feature extraction. 


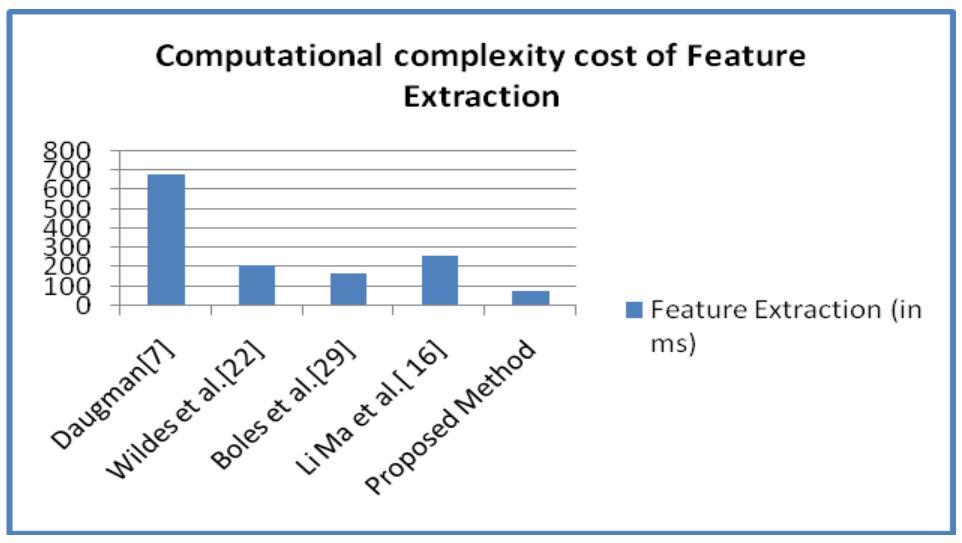

Figure 12. Comparative Graph

Table 4. Computational Complexity Cost for Feature Extraction

\begin{tabular}{cc}
\hline Methods & $\begin{array}{c}\text { Feature Extraction (in } \\
\mathrm{ms} \text { ) }\end{array}$ \\
\hline Daugman[7] & 682.5 \\
Wildes et al.[22] & 210.0 \\
Boles et al.[29] & 170.3 \\
Li Ma et al.[ 16] & 260.2 \\
Proposed Method & 78.0 \\
\hline
\end{tabular}

\subsection{Matching}

Hamming distance is beneficiary as it performs XOR operation on Boolean vectors.

Step 1: Compare Query image feature vector with stored image feature vector of database.

Step 2: Hamming Distance is calculated for each image feature vector.

Step 3: Finally Calculate minimum Hamming Distance.

If Hamming Distance between two feature vectors is greater, the difference between them is also greater. Two similar irises will fail the test since the difference between them will be small. The Hamming Distance (HD) between two Boolean vectors is defined (as in eq (6)).

$$
\mathrm{HD}=\frac{1}{N} \sum_{j=1}^{N} C_{A}(j) \oplus C_{B}(i)
$$

Where $C_{A}$ and $C_{B}$ are the coefficients of two iris images, $N$ is the size of the feature vector, Ex-OR is the Boolean operator that gives a binary 1 if the bits at the position $\mathrm{j}$ in $\mathrm{C}_{\mathrm{A}}, \mathrm{C}_{\mathrm{B}}$ are different and 0 if they are similar. Daugman [9] conducted tests on very large number of iris patterns, i.e., up to 200 Billion irises images and resulted that the maximum Hamming distance that exists between two irises belonging to the same person is 0.32 .

- If $\mathrm{HD}<=$ Threshold then Match successful.

- If HD> Threshold then Match unsuccessful i.e. different person or left and right eye iris of the same person. 


\section{Results and Discussion}

\subsection{Results}

Experiment results are evaluated based on parameters such as False Acceptance Rate (FAR), False rejection rate(FRR), Equal Error rate(EER) and Correct recognition rate(CRR). Number of times an unauthenticated person accepted by system is FAR; number of time an authentic person is rejected by the system is FRR. The point where FAR and FRR meets is EER, smaller the EER more accurate system performance, i.e., CRR. Our results are very encouraging in terms of reduced EER and Increased CRR using scanning method and five levels decomposition technique. The training image consider are 756 iris images and testing images are 100 as in Figure 14. Table 5 gives the comparision of Haar, db2 and db4 in terms of Feature vector, EER, FAR, FRR and shows that haar is best suited for efficient feature extraction algorithm. The implementation process is by using wavelets such as Haar, db2, db4 algorithms and the frequency distribution for HD is calculated which is as shown in Figure 15(c), score distribution of intraclass and interclass hamming distance for imposter and genuine of the system is calculated and graph as shown in Figure 15 (a) and (b) and Figure 14 shows overall score distribution of the system with mean of 0.38 and sigma of 0.08 . Our system results are quite encouraging with false Non match rate of $0.025 \%$ and false match rate of $0.033 \%$ for Haar wavelet with different hamming distance. HD distribution for intra class and interclass overlap each other to get the separation between them which requires FAR and FRR, if smaller HD then FAR reduces and FRR increase and if HD increases then FAR increases and FRR decreases as illustrated in Figure 16. The ROC curve for Haar is as shown in Figure 17.

The FAR and FRR is calculated for the system for different HD using the formula as in eq 7 and eq 8
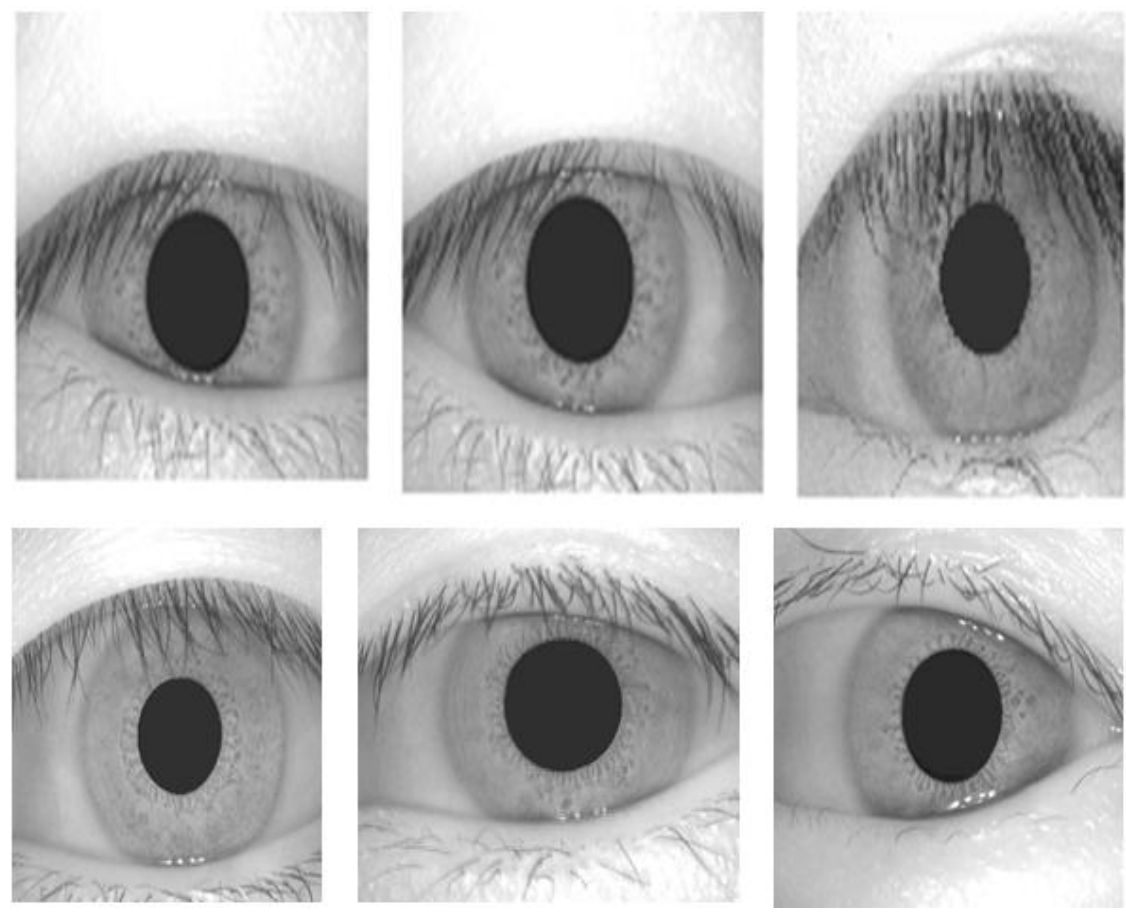

Figure 13. Some CASIA V1 Iris Testing Images 
International Journal of Signal Processing, Image Processing and Pattern Recognition Vol.6, No.6 (2013)

Table 5. Wavelet Algorithm with Threshold and other Performance Parameter

\begin{tabular}{|l|l|l|l|l|l|l|}
\hline Algorithm & Threshold & FAR \% & FRR\% & EER & CRR\% & $\begin{array}{l}\text { Feature } \\
\text { Vector } \\
\text { elements }\end{array}$ \\
\hline Haar & 0.32 & $0.033 \%$ & $0.025 \%$ & $0.028 \%$ & $99.97 \%$ & 90 \\
\hline Db2 & 0.32 & $0.18 \%$ & $0.69 \%$ & $0.36 \%$ & $99.64 \%$ & 240 \\
& & & & & & \\
\hline Db4 & 0.4 & $0.03 \%$ & $0.40 \%$ & $0.22 \%$ & $99.78 \%$ & 660 \\
\hline
\end{tabular}

$$
\begin{aligned}
\mathrm{FAR} & =\frac{\text { No.of times different persons match }}{\text { No.of comparissions between different persons }} * 100 \\
\mathrm{FRR} & =\frac{\text { No.of times persons rejected }}{\text { No.of comparissions between same persons }} * 100
\end{aligned}
$$

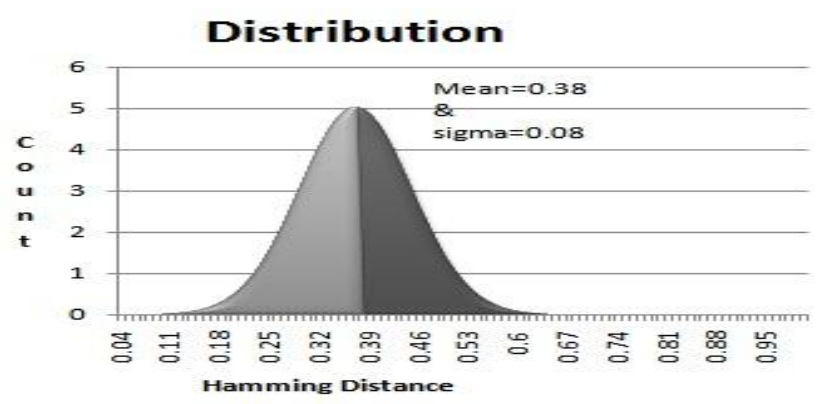

Figure 14. Score Distribution with Mean=0.38 \& Sigma=0.08

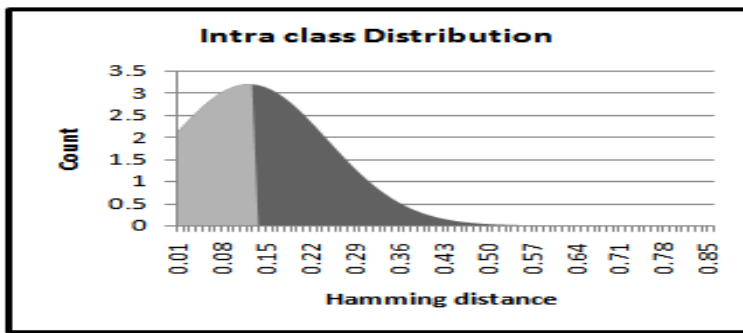

(a)

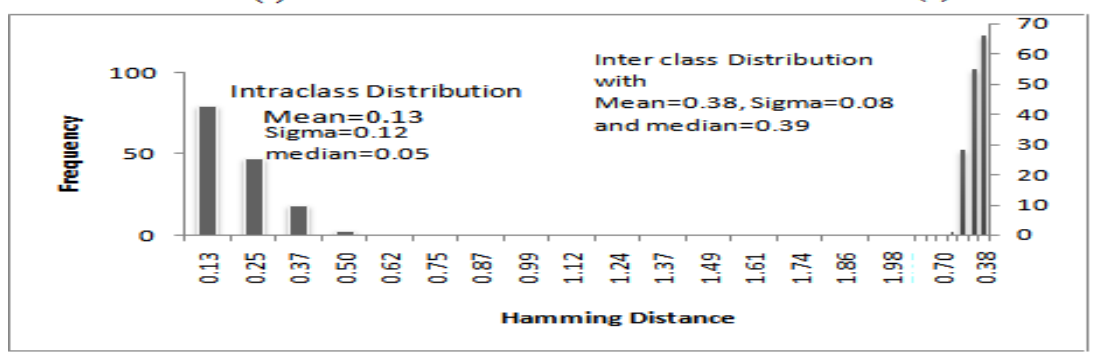

(C)

(b)

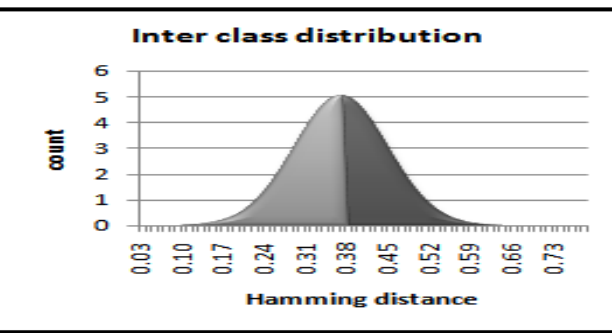

(a) \& (b) Score Distribution of Inter and Intra Class and (c) Frequency Distribution of Inter and Intra Class 


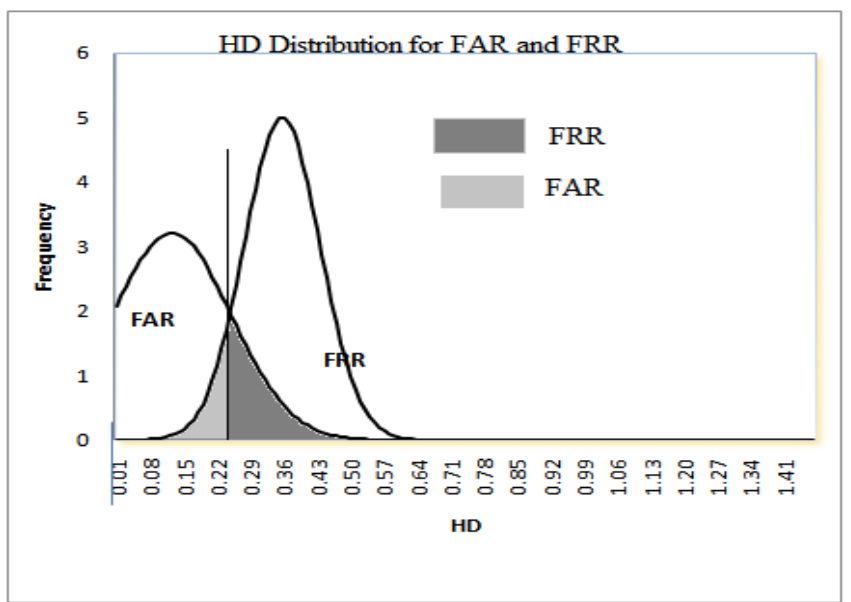

Figure 16. Score Distribution for Imposter and Genuine for Different Hamming Distance

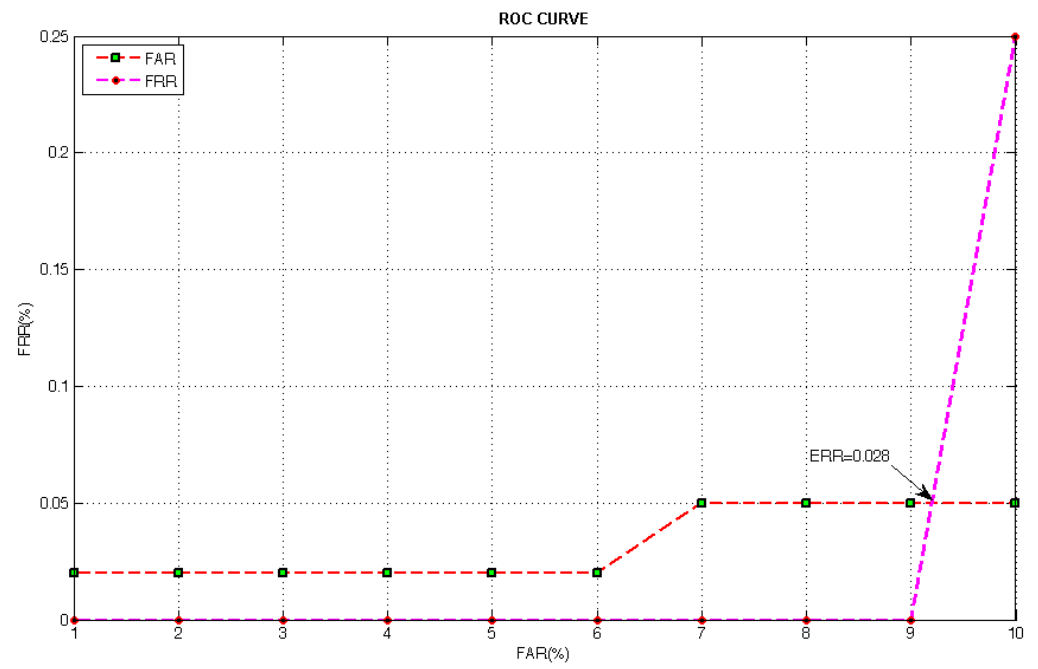

Figure 17. Roc Curve for Haar

\subsection{Comparision and Discussion}

Our method is using CASIA Iris database for verification and identification modes and found that our results are also encouraging in terms of accuracy, efficiency and reduced computational complexity. Results are compared with published result of methods $[1,4,5$, 16, 22, and 29]. Table 14 and Figure 18 gives the comparison in terms of CRR and EER. Table 5 shows Daugman [7] method achieves 98.60\% CRR with EER of 0.08, Wildes [22] achieves much better accuracy as compared to [7] but lacks with EER of 1.76. Proposed method shows encouraging results with reduced EER of 0.0288 and recognition rate of CRR is 99.97 and EER achieved by our system is less than methods proposed by [1, 7, 16, 22, and 29]. 


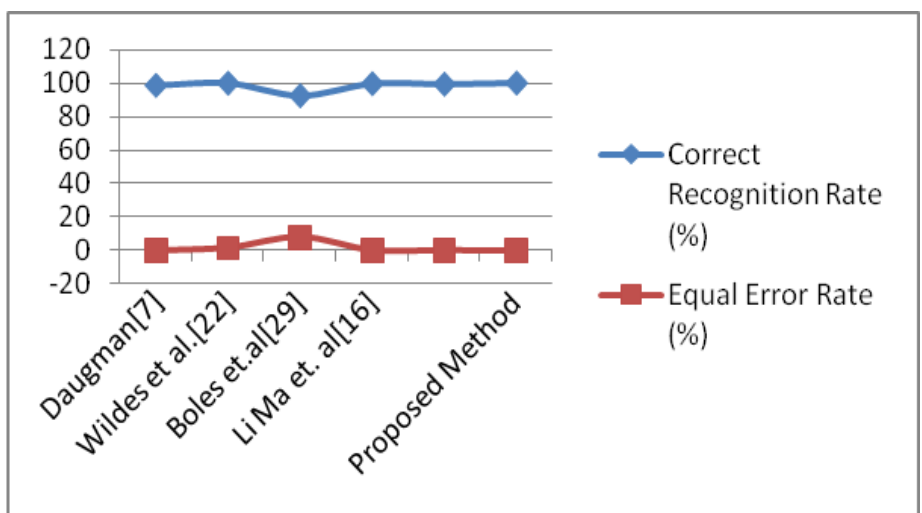

Figure 18. Comparision of CRR and EER

Table 6. Comparision of CRR and EER

\begin{tabular}{ccc}
\hline Methods & Correct Recognition Rate (\%) & Equal Error Rate (\%) \\
\hline Daugman[7] & 98.60 & 0.08 \\
Wildes et al., [22] & 99.90 & 1.76 \\
Boles et al., [29] & 92.64 & 8.13 \\
Li Ma et al.,[16] & 99.60 & 0.29 \\
Poursaberi and Araabi[1] & 99.31 & 0.2687 \\
Proposed Method & 99.97 & 0.03 \\
\hline
\end{tabular}

\section{Conclusion and Future Work}

\subsection{Conclusion}

Enhancing iris recognition algorithm based on Haar wavelet with quality texture features of iris within feature vector, even though obstruction of eyelashes and eyelids and our proposed method also works perfect for narrowed eyelid as proposed method consider small part of the iris even though it is occluded. So, it increases the overall accuracy of the system with less computational cost in terms of time as compared with methods of other authors and high recognition rate with reduced EER, FAR, FRR. The results also show the performance evaluation with different parameters with different class of variations i.e., Inter class hamming distance variation and Intra class hamming distance variation.

\subsection{Future Work}

Our experimental results demonstrates that enhanced method for pupil extraction and five level decomposition for iris image has significantly encouraging and promising results in terms of EER and CRR. Our Feature work will include:

- Improving effectiveness in matching in terms of computational cost time.

- We are also currently working on global textural analysis with more levels of decomposition with accurate feature

- Extraction of feature from larger database similar to Daugman's methods. 


\section{Acknowledgements}

We are sincerely thankful to Institute of Automation Chinese Academy of science for providing CASIA database to do our research work, also shows gratitude to renowned authors for their work in field of Iris biometric for providing results.

\section{References}

[1] P. and H. N. Araabi, "Iris Recognition for Partially Occluded Methodology and Sensitivity Analysis", EURASIP journal on Advances in Signal processing, vol. 2007, (2005), Article ID 36751.

[2] A. Poursaberi and B. N. Araabi (2005), "A Half Eye Wavelet Based for Iris Recognition", proceeding of the 2005, 5th International Conference on Intelligent Systems Design and Applications (ISDA’05).

[3] A. D. Rahulkar and R. S. Holambe, "Half-Iris Feature Extraction and Recognition using a New Class of Biorthogonal triplet Half-Band Filter Bank and Flexible k-out-of-n: A postclassifier", IEEE Transactions on Information Forensics and Security, vol. 7, no. 1, (2012) February.

[4] J. Daugman, "How Iris Recognition works", proceedings of 2002 International conference on Image processing, vol. 1, (2002).

[5] J. Daugman, "High confidence visual recognition of persons by a Test of statistical Independence", IEEE Transactions on pattern analysis and machine intelligence, vol. 15, no. 11, (1993), pp. 1148-1161.

[6] J. Daugman, "How Iris Recognition works, IEEE Transactions on Circuits and systems for video Technology", vol. 14, no. 1, (2004) January.

[7] J. Daugman, "Statistical Richness of Visual Phase Information: Update on recognizing persons by Iris patterns", International Journal of computer vision, vol. 45, no. 1, (2001), pp. 25-38.

[8] J. M. H. Ali and A. Ella Hassanien, "An Iris Recognition System to Enhance E-security Environment Based on Wavelet Theory", AMO-Advance Modeling and Optimization, vol. 5, no. 2, (2003).

[9] J. Daugman, "Probing the Uniqueness and Randomness of IrisCodes: Results from 200 Billion Iris Pair Comparisons", Proceedings of the IEEE, vol. 94, no. 11, (2006) November, pp. 1927-1935.

[10] K. Karu, K. Anil Jain and R. M. Bolle, "Is there any texture in the Image?", IEEE Transactions on Pattern Recognition, vol. 29, no. 9, (1996), pp. 1437-1446.

[11] K. W. Bowyer, K. Hollingsworth and P. J. Flynn, "Image understanding for iris biometrics: A survey", Elsevier computer vision and Image Understanding, vol. 110, (2008), pp. 281-307.

[12] L. Ma, Y. Wang and T. Tan, "Iris recognition Based on multichannel Gabor Filtering", Proc. of the $5^{\text {th }}$ Asian conference on computer vision, vol. I, (2002), pp. 279-283.

[13] L. Ma, Y. Wang and T. Tan, "Iris Recognition using Circular Symmetric Filters", proc. of the $16^{\text {th }}$ International Conference on pattern recognition, vol. II, (2002), pp.414-417.

[14] L. Ma, T. Yunhong Wang and D. Zhang, "Personal identification based on iris texture analysis", IEEE Transactions on Pattern Analysis and machine Intelligence, vol. 25, no. 12, (2003).

[15] L. Ma, T. Tan, Y. Wang and D. Zhang, "Efficient Iris Recognition by Characterizing Key Local Variations", IEEE Transactions on Image processing, vol. 13, no. 6, (2004) June.

[16] L. Ma, "Local Intensity Variation Analysis for Iris Recognition", Elsevier- Pattern Recognition, vol. 37, no. 6, (2004), pp. 1287-1298.

[17] M. Nabti, L. Ghouti and A. Bouridane, "An effective and fast iris recognition system based on a combined multiscale feature extraction technique", Elsevier-Pattern Recognition, vol. 41, (2008), pp. 868-879.

[18] M. Vatsa, R. Singh and A. Noore, "Improving Iris recognition performance using segmentation, Quality Enhancement", Match Score Fusion, and Indexing, IEEE Transactions on Systems, Man, and Cybernetics B, Cybern., vol. 38, no. 4, pp. 1021-1034.

[19] R. Islam, W. Yin Chai and A. Khatun, "Partial Iris Image Recognition Using Wavelet Based Texture Features", International Conference on Intelligent and Advance Systems (ICIAS), (2010).

[20] J. Mao and A. K. Jain, "Artificial neural network for feature extraction and multivariate data projection", IEEE Transactions on neural network, vol. 6, no. 2, (1995) March, pp. 296-317.

[21] R. P. Wildes, "Iris Recognition: An Emerging Biometric Technology", Proceeding of the IEEE, vol. 85, (1999), pp 1348-1363.

[22] R. Wildes, J. Asmuth, G. Green, S. Hsu, R. Kolczynski, J. Matey and S. McBride, “A machine-vision system for Iris Recognition”, Machine vision and Applications, vol. 9, (1996), pp. 1-8.

[23] R. Yew Fatt Ng, Y. Hour Tay and K. Ming Mok, "A Review if Iris Recognition Algorithm”, IEEE Transaction, DOI 978-1-4244-2328-6/08, (2007).

[24] R. W. Ives, A. J. Guidry and D. M. Etter, "Iris Recognition using Histogram Analysis", IEEE conference on signal, system and computer, (2004), pp. 562-566. 
International Journal of Signal Processing, Image Processing and Pattern Recognition

Vol.6, No.6 (2013)

[25] S. Lim, K. Lee, O. Byeon and T. Kim, "Efficient Iris Recognition through Improvement of Feature Vector and Classifier", ETRI Journal, vol. 23, no. 2, (2001) June, pp. 61-70.

[26] S. Mallat and W. Hwang, "singularity detection and processing with wavelet", IEEE Transaction on information Theory, vol. 38, no. 2, (1992), pp. 617-643.

[27] S. M. Elsherief, M. E. Allam and M. W. Fakhr, "Biometric Personal identification Based on Iris Recogition", International conference on computer Engineering and Systems, (2006) November, pp. 208-213, 5-7.

[28] W. Boles and B. Boashash, "A Human Identification Technique using Images of the Iris and wavelet transform”, IEEE Transactions on signal processing, vol. 46, no. 4, (1998) April.

[29] http://www.sinobiometrics.com/resources.htm. 